aber zugleich durch die verschiedenen Entscheidungsoptionen und Handlungsläufe nicht zugunsten direkter Demokratie entmachten. Zudem würden eher heikle Aspekte wie Quoren oder Themenausschlüsse durch die Verfahrenskonstruktion von vornherein vermieden. Klar sollte sein, dass ein obligatorisches Referendum kein zusätzlicher Input-Kanal sein, aber immerhin die vorhandenen Kanäle vielleicht freihalten kann. Wie die Bürger dann abstimmen werden, ist aber andere Frage.

\title{
Raucher oder Nichtraucher - ist das die Frage? Wahlbeteiligung und Abstimmungsverhalten beim Volksentscheid über das Rauchverbot in Bayern
}

\author{
Harald Schoen, Alexander Glantz und Rebecca Teusch
}

\section{Nichtraucherschutz in Bayern - eine unendliche Geschichte?}

Am 4. Juli 2010 waren knapp 9,4 Millionen Bayern dazu aufgerufen, in einem Volksentscheid über eine Verschärfung des Nichtraucherschutzes zu entscheiden. Damit fand eine längere Auseinandersetzung über das Rauchen im öffentlichen Raum, vor allem aber in Gaststätten, ihr - vorläufiges - Ende. ${ }^{1}$ Im Dezember 2007 beschloss der Bayerische Landtag das bis dahin bundesweit strikteste Rauchverbot. Das einzige Schlupfloch, das das Gesetz gelassen hatte, nutzten etliche Raucher und richteten bayernweit Raucherclubs ein. Nach der Landtagswahl 2008, bei der die CSU die absolute Mehrheit verloren hatte ${ }^{2}$, wurde das Rauchverbot gelockert. Die neue Landtagsmehrheit aus CSU und FDP führte verschiedene Ausnahmen beim Nichtraucherschutz ein, unter anderem für kleine Kneipen, Raucherräume und Festzelte. ${ }^{3}$ Auf diese Entscheidung hin initiierte die ÖDP mit Nichtraucherverbänden und Ärzten eine Unterschriftensammlung, um ein Volksbegehren „Für echten Nichtraucherschutz!“ zugunsten eines ausnahmslosen Rauchverbots in der Gastronomie einzuleiten. Dies gelang, und ebenso war das Volksbegehren erfolgreich, da vom 19. November bis zum 2. Dezember 2009 knapp 14 Prozent der Stimmberechtigten - und damit deutlich mehr als die erforderlichen zehn Prozent - eine Unterschrift zugunsten des Begehrens leisteten. Der Landtag lehnte daraufhin mit den Stimmen von CSU und FDP den Gesetzentwurf des Volksbegehrens ab und ebnete damit den Weg für einen Volksentscheid.

1 Vgl. zur Vorgeschichte des Volksbegehrens Bärbel Weixner, Nichtraucherschutz in Bayern - der Weg eines erfolgreichen Volksbegehrens und Volksentscheids im Freistaat, in: Lars P. Feld / Peter M. Huber / Otmar Jung / Christian Welzel / Fabian Wittreck (Hrsg.), Jahrbuch für direkte Demokratie 2010, Baden-Baden 2011, S. 255 - 294.

2 Vgl. dazu Rainer-Olaf Schultze / Jan Grasnick, Die bayerische Landtagswahl vom 28. September 2008: Betriebsunfall oder Ende eines Mythos?, in: ZParl, 40. Jg. (2009), H. 1, S. 34 - 55.

3 Vgl. Gesetz zum Schutz der Gesundheit (Gesundheitsschutzgesetz - GSG), Bayerisches Gesetzund Verordnungsblatt (GVBl), München 2009, S. 384. 
Den engagierten, aber ressourcenschwachen Abstimmungskampf vor dem Volksentscheid bestritten im Wesentlichen zwei Gruppen. Das Bündnis „Bayern atmet auf - Ja zum Nichtraucherschutz" warb mit der Unterstützung von ÖDP, SPD und Bündnis 90/Die Grünen für ein komplettes Rauchverbot in Gaststätten, um die Menschen vor den Gefahren des Passivrauchens zu schützen. Die Nein-Seite gründete mit dem Slogan „Bayern sagt nein“ das „Aktionsbündnis für Freiheit und Toleranz“ und fand die Unterstützung der FDP. Dieses Bündnis versuchte sich nicht nur zum Anwalt der Raucher, sondern des bayerischen „Leben und leben lassen“ zu machen. Damit versuchte es der verbreiteten Darstellung und Wahrnehmung, beim Volksentscheid über das Rauchverbot handele es sich um einen Konflikt zwischen Rauchern und Nichtrauchern ${ }^{4}$, entgegenzuwirken.

Dem Ruf an die Wahlurnen folgten am 4. Juli 2010 schließlich 37,7 Prozent der Stimmberechtigten. Sie sorgten für ein deutliches Ergebnis: 61 Prozent der Stimmen wurden für den Gesetzentwurf „Für echten Nichtraucherschutz!“ abgegeben, 39 Prozent sprachen sich für die bis dahin geltende Regelung aus. ${ }^{5}$ So konnten in Bayern zum 1. August 2010 die bundesweit striktesten Regeln zum Nichtraucherschutz in Kraft treten. Sie sehen unter anderem ein absolutes Rauchverbot in Kultur-, Freizeit-, Kinder- und Jugendeinrichtungen sowie in Sport- und Gaststätten vor. Die bislang bestehenden Ausnahmeregelungen in der Gastronomie wurden ersatzlos gestrichen. ${ }^{6}$

Die deutliche Mehrheit für den Gesetzentwurf wurde in der Öffentlichkeit nicht selten als „Triumph der Nichtraucher"7 wahrgenommen und mit Überraschung kommentiert. ${ }^{8}$ Interpretiert man den Volksentscheid als Auseinandersetzung zwischen Rauchern und Nichtrauchern, erscheint die Überraschung über den Ausgang erstaunlich. ${ }^{9}$ Bayerns Bevölkerung umfasst gut 70 Prozent Nichtraucher und weniger als 30 Prozent Raucher. ${ }^{10}$ Nimmt man an, allein das Rauchverhalten entscheide über das Stimmverhalten, könnte alles überraschen - nur nicht ein klarer Sieg der Ja-Seite. Die Gegner des strikten Rauchverbots erkannten dieses Problem offenbar frühzeitig und suchten - wie oben skizziert - die Auseinandersetzung gerade nicht als Konflikt zwischen Rauchern und Nichtrauchern, sondern als Wahl zwischen individueller Freiheit und staatlicher Intervention darzustellen. Auf

4 Vgl. etwa Abendzeitung vom 15. April 2010, S. 10.

$5 \mathrm{Zu}$ den Ergebnissen siehe http://www.volksentscheid2010.bayern.de (Abruf am 25. August 2011).

6 In bestimmten öffentlichen Gebäuden und Einrichtungen besteht jedoch die Möglichkeit, Raucherräume einzurichten. Vgl. Gesetz zum Schutz der Gesundheit (Gesundheitsschutzgesetz GSG), Bayerisches Gesetz- und Verordnungsblatt (GVBl), München 2010, S. 314.

7 Vgl. Katja Auer / Peter Fahrenholz / Mike Szymanski, Triumph der Nichtraucher, in: SüddeutscheOnline vom 4. Juli 2010, http://www.sueddeutsche.de/bayern/volksentscheid-in-bayern-triumph-der-nichtraucher-1.969874 (Abruf am 25. August 2011).

8 Vgl. etwa „61 Prozent für totales Rauchverbot“, ARD-Mittagsmagazin vom 5. Juli 2010, http:// www.br-online.de/mittagsmagazin/0507_rauchverbot.shtml (Abruf am 25. August 2011).

9 Zur Überraschung mag auch eine kurz vor dem 4. Juli 2010 veröffentlichte Meinungsumfrage beigetragen haben, die einen unzutreffenden Eindruck von den Mehrheitsverhältnissen vermittelte. Siehe dazu auch Harald Schoen, Das Kopf-an-Kopf-Rennen, das nie eines war, http://blog.zeit. de/politik-nach-zahlen/2010/07/08/das-kopf-an-kopf-rennen-das-nie-eines-war_2448 (Abruf am 25. August 2011).

10 Vgl. Bayerisches Staatsministerium für Umwelt und Gesundheit, Gesund in Bayern, München 2010, S. 35. Die Angaben basieren auf dem Mikrozensus 2005 und beziehen sich auf die Bevölkerung ab 15 Jahren. 
diese Weise sollten auch Nichtraucher für die Nein-Seite gewonnen werden, um zu einer Mehrheit gegen den Gesetzentwurf für ein striktes Rauchverbot beizutragen.

Es bleibt zu klären, welche Rolle das Rauchverhalten tatsächlich bei der Abstimmung über das Rauchverbot in Bayern spielte. Dieser Frage soll nachgegangen werden auf der Grundlage von Daten aus einer telefonischen Befragung 4.000 zufällig ausgewählter Stimmberechtigter, die im Rahmen des DFG-Projekts „Politische Urteilsbildung in direktdemokratischen Verfahren. Eine Analyse zum Volksentscheid über den Nichtraucherschutz in Bayern 2010" vom 25. Mai bis zum 3. Juli 2010 durchgeführt wurde. ${ }^{11}$

\section{Kampagnendynamik und politische Mobilisierung}

Die Kampagnen der Befürworter und Gegner eines strikten Rauchverbots in Bayern zielten unter anderem darauf ab, bei Bürgern Interesse am Volksentscheid zu wecken und sie zur Stimmabgabe zu bewegen. Alles in allem deuten die von uns erhobenen Daten darauf hin, dass die Kampagne bis etwa eine Woche vor dem Abstimmungstag kaum auf Resonanz gestoßen ist, und zwar bei Rauchern und Nichtrauchern in ähnlichem Maße. Gaben Ende Mai rund zehn Prozent der Raucher und acht Prozent der Nichtraucher an, in verschiedenen Medien Werbung zum Volksentscheid gesehen zu haben, so waren es in der Woche vor der Abstimmung rund 31 Prozent der Raucher und 39 Prozent der Nichtraucher. Der Anteil derjenigen, die Flugblätter oder ähnliches Material gelesen hatten, stieg bei Rauchern von neun auf etwa 25 Prozent und bei Nichtrauchern von vier auf 16 Prozent. Plakate der Pro- und Contra-Seite hatten in der Woche vor der Abstimmung 65 Prozent der Raucher und 57 Prozent der Nichtraucher gesehen (nicht tabellarisch oder graphisch ausgewiesen). Die Kampagne entwickelte sich also durchaus dynamisch, hat aber beileibe nicht alle Bürger erreicht. Zudem lassen sich zwischen Rauchern und Nichtrauchern keine konsistenten und gravierenden Unterschiede in der Rezeption erkennen.

Die Kampagnenkontakte scheinen nicht wesentlich dazu beigetragen zu haben, dass sich Bürger für den Volksentscheid interessierten und sich damit auseinandersetzten. Ablesen lässt sich das etwa daran, dass das Interesse an dem Volksentscheid im Verlauf der Kampagne kaum zunahm (siehe Abbildung 1). ${ }^{12}$ Waren Ende Mai nur etwa 33 Prozent der Raucher und 41 Prozent der Nichtraucher an dem Abstimmungskampf der beiden Kontrahenten (sehr) interessiert, bekundeten zum Ende immerhin rund 41 Prozent der Raucher und jeder zweite Nichtraucher sein Interesse. Fasst man den gesamten Zeitraum zusammen, so gaben etwa 45 Prozent der Nichtraucher und 39 Prozent der Raucher an, sich stark oder

11 Die Daten wurden im Rolling-Cross-Section-Design erhoben. Bei diesem Verfahren wird eine Zufallsstichprobe von Befragten so über den Erhebungszeitraum verteilt, dass die Respondenten jedes Tages als eine repräsentative Stichprobe aus der Grundgesamtheit betrachtet werden können. Siehe zur Analyse dieses Datentyps Richard Johnston / Henry E. Brady, The Rolling CrossSection Design, in: Electoral Studies, 21. Jg. (2002), H. 2, S. 283 - 295; Rüdiger Schmitt-Beck I Thorsten Faas / Christian Holst, Der Rolling Cross-Section Survey - Ein Instrument zur Analyse dynamischer Prozesse der Einstellungsentwicklung: Bericht zur ersten deutschen RCS-Studie anlässlich der Bundestagswahl 2005, in: ZUMA-Nachrichten, 58. Jg. (2006), S. 13 - 49.

12 Für die graphischen Darstellungen wurden die Daten mit Hilfe des LOWESS-Verfahrens geglättet, um übergreifende Trends besser zu veranschaulichen. Vgl. hierzu Rüdiger Schmitt-Beck / Thorsten Faas / Christian Holst, a.a.O. (Fn. 11). 


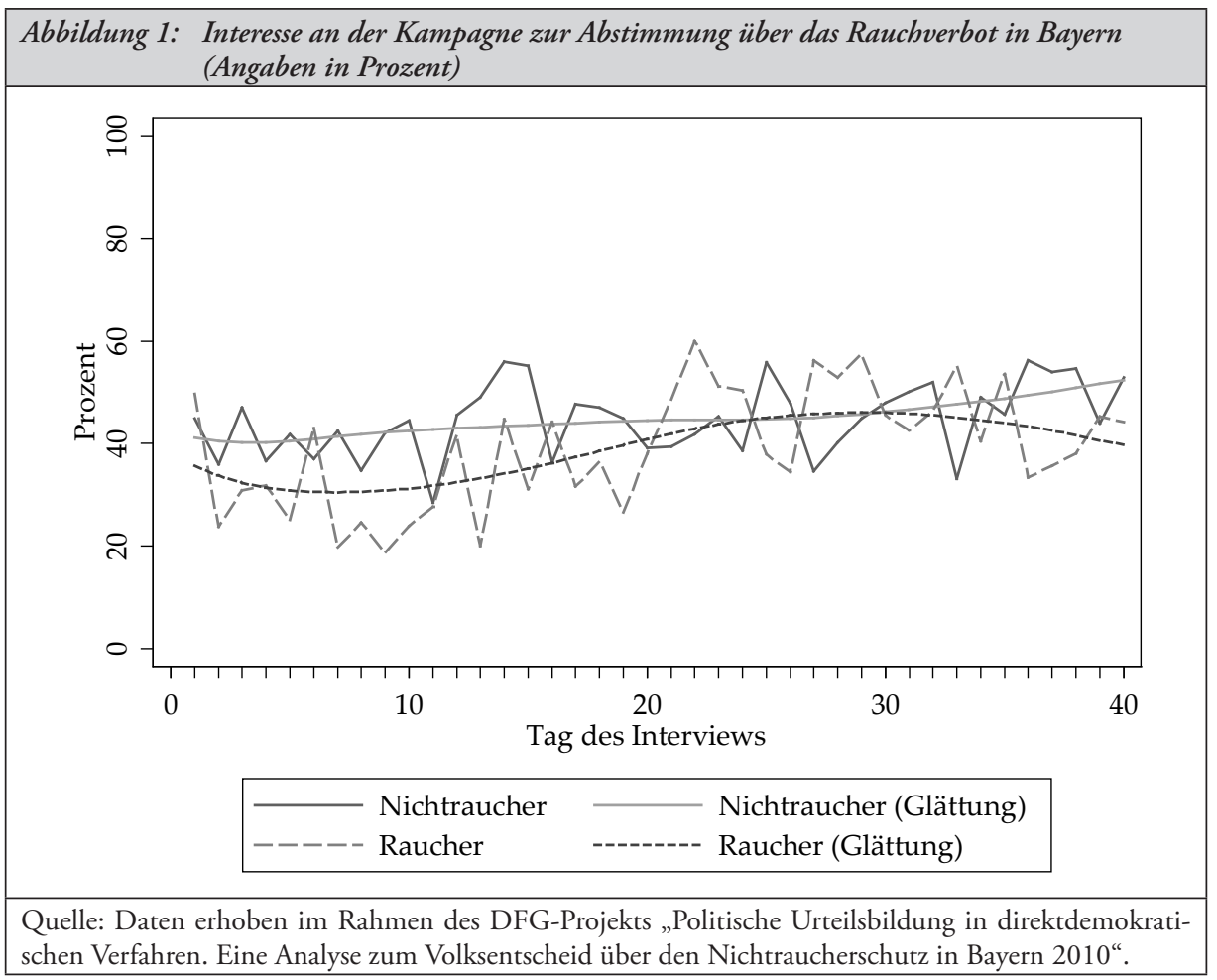

sehr stark für die Kampagne zu interessieren. Insgesamt lassen sich somit nur geringe Unterschiede zwischen Rauchern und Nichtrauchern erkennen.

Das relativ geringe Interesse findet seine Entsprechung in der Informiertheit der Bürger. Ende Mai hielt sich jeder fünfte Befragte für subjektiv (sehr) gut informiert über den Volksentscheid, eine Woche vor der Abstimmung waren es rund 40 Prozent. Gleichzeitig hat das objektive Wissen über den Volksentscheid nur vereinzelt zugenommen. Hatten anfangs nur sechs Prozent der Nichtraucher und zwölf Prozent der Raucher gewusst, wann der Volksentscheid stattfinden wird, kannten eine Woche vor der Abstimmung 78 Prozent der Nichtraucher und 69 Prozent der Raucher den Termin. Das inhaltliche Wissen über den Volksentscheid wurde mit fünf Fragen zu Regelungen des Gesundheitsschutzgesetzes und des Abstimmungsverfahrens gemessen. ${ }^{13}$ Im Durchschnitt konnten sowohl Raucher als auch Nichtraucher 2,4 Fragen richtig beantworten. Die Kenntnisse sind somit unabhängig

13 Die Befragten wurden gebeten anzugeben, ob die folgenden Aussagen zum Gesetzentwurf ihrer Meinung nach zuträfen oder nicht: „Der Gesetzentwurf verbietet Rauchen in Bierzelten“, „Bei mehrmaliger Übertretung des Rauchverbots sieht der Gesetzentwurf eine Haftstrafe von bis zu 12 Monaten vor“, „Nach dem Gesetzentwurf können in Krankenhäusern Raucherräume eingerichtet werden“, „Wenn in einer Gaststätte geraucht wird, kann nach dem Gesetzentwurf der Raucher bestraft werden, nicht aber der Betreiber der Gaststätte“, „Wenn jemand verhindern möchte, dass der Gesetzentwurf in Kraft tritt, dann sollte er dagegen stimmen oder nicht an der Abstimmung teilnehmen." Auf dieser Grundlage wurde ein Zählindex richtiger Antworten gebildet, der von 0 bis 5 reicht. 


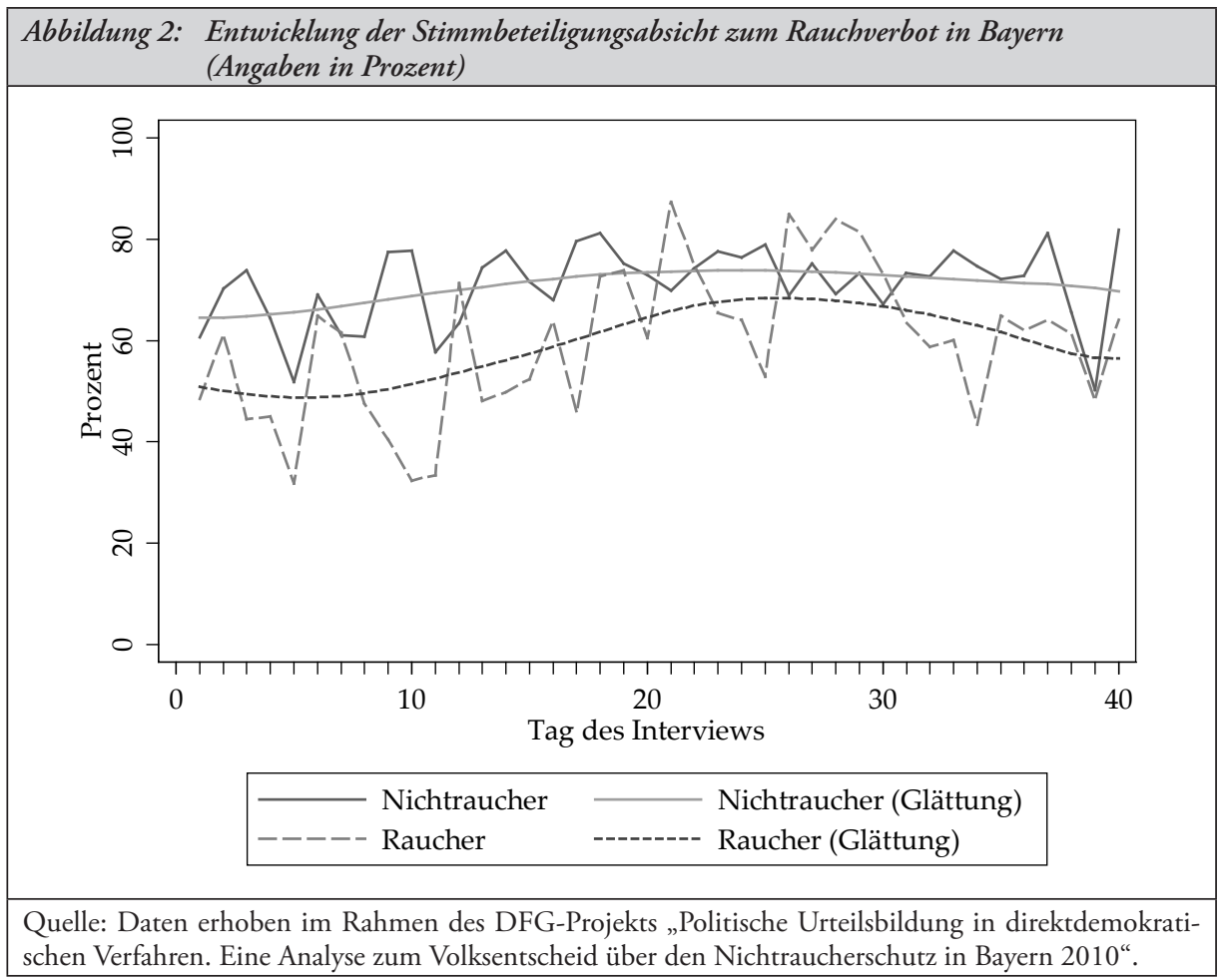

vom Rauchverhalten, was in den vierzig Tagen bis zum Volksentscheid so blieb, denn während des gesamten Untersuchungszeitraums sind kaum Veränderungen im Informationsniveau von Rauchern und Nichtrauchern erkennbar. Die Kampagnen von Befürwortern und Gegnern konnten hier also kaum Aufklärungserfolge erzielen.

Spärlicher als die inhaltlichen Kenntnisse war das Wissen über die zentralen Akteure und ihre Positionen. In der letzten Woche der Kampagne wussten jeweils rund zehn Prozent der Raucher und Nichtraucher, dass Sebastian Frankenberger, der führende Kopf der Ja-Seite, für den Gesetzentwurf „Für echten Nichtraucherschutz!" eintrat. Kaum besser war es um das Wissen von Rauchern und Nichtrauchern über die Position des „Aktionsbündnis Freiheit und Toleranz" bestellt. Auf niedrigem Niveau lassen sich somit auch in dieser Hinsicht keine Unterschiede zwischen Rauchern und Nichtrauchern erkennen.

Etwas anders verhält es sich bei der Bereitschaft, am Volksentscheid teilzunehmen. In Abbildung 2 fällt zunächst auf, dass der Anteil derjenigen Befragten, die angaben, bestimmt oder wahrscheinlich ihre Stimme abzugeben, deutlich über der tatsächlichen Beteiligungsrate von 37,7 Prozent liegt. Darin kommen wohlbekannte Probleme der Umfrageforschung bei der Messung politischen Verhaltens zum Ausdruck. ${ }^{14}$ Unter bestimmten Annahmen

14 Dabei handelt es sich einerseits um Stichprobenprobleme, das heißt politisch aktive Menschen nehmen wahrscheinlicher an Befragungen teil, andererseits um Messfehler, das heißt nicht-aktive Befragte geben an, politisch aktiv zu sein. Siehe hierzu beispielsweise Robert Bernstein / Anita Chadha I Robert Montjoy, Overreporting Voting: Why it Happens and Why it Matters, in: Public 
sollten sie den Vergleich zwischen Rauchern und Nichtrauchern aber nicht wesentlich verzerren. ${ }^{15}$ Während sich 71 Prozent der Nichtraucher an der Abstimmung beteiligen wollten, waren die Raucher mit 59 Prozent deutlich weniger geneigt, ihre Stimme abzugeben. Wie man in Abbildung 2 erkennt, näherten sich Raucher zwar zeitweise den Nichtrauchern an, aber im Großen und Ganzen blieben die Unterschiede im Laufe der Kampagne relativ stabil.

Zwischen Rauchern und Nichtrauchern bestanden also weder größere Unterschiede im Interesse am Volksentscheid noch in den Kenntnissen über zentrale Inhalte des Gesetzentwurfs. Allerdings zeigen Nichtraucher eine deutlich höhere Bereitschaft als Raucher, sich an dem Volksentscheid zu beteiligen. Unter der Annahme, dass das Rauchverhalten bei der Entscheidung für oder wider die Verschärfung des Rauchverbots den Ausschlag gab, machten es diese Mobilisierungsunterschiede für die Nein-Seite noch schwerer.

\section{Rauchverhalten und Stimmentscheidung}

Raucher und Nichtraucher entschieden sich beim Volksentscheid am 4. Juli sehr unterschiedlich. Wie Abbildung 3 zeigt, beabsichtigten im gesamten Untersuchungszeitraum rund 80 Prozent der Nichtraucher, für das strikte Gesundheitsschutzgesetz zu stimmen. Auf Seiten der Raucher lag die Zustimmungsrate mit 28 Prozent deutlich niedriger. Das Stimmverhalten ist somit deutlich entlang der Unterscheidung zwischen Rauchern und Nichtrauchern polarisiert - und entspricht insoweit den Wahrnehmungen und Erwartungen der Öffentlichkeit.

Außerdem blieb der Anteil der Nichtraucher, die sich für ein Ja entscheiden wollten, in den letzten 40 Tagen vor dem Volksentscheid weitgehend konstant. Dagegen gelang es der Nein-Seite offenbar im Laufe der Kampagne, die Bereitschaft der Raucher zu steigern, am 4. Juli mit Nein zu votieren. Diese stimmten also zunehmend entlang der erwarteten Scheidelinie. Daraus resultierte eine gewisse weitere Polarisierung des Stimmverhaltens zwischen Rauchern und Nichtrauchern. Ließ sich das Votum eines Bürgers bereits zu Beginn des Untersuchungszeitraums relativ gut aus einer Information über seinen Tabakkonsum ableiten, so gelang dies im Kampagnenverlauf noch besser.

Diese deutliche Polarisierung im Stimmverhalten deutet darauf hin, dass die Kampagnenstrategie der Nein-Seite nicht aufgegangen ist. Die Gegner des verschärften Nichtraucherschutzes hatten das Ziel verfolgt, möglichst viele Nichtraucher auf ihre Seite zu ziehen, indem sie den Volksentscheid gerade nicht als Konflikt zwischen Rauchern und Nichtrauchern darzustellen suchten. Vielmehr sollten die Stimmberechtigten den Eindruck gewin-

Opinion Quarterly, 65. Jg. (2001), H. 1, S. 22 - 44; Roger Tourangeau / Robert M. Groves / Cleo D. Redline, Sensitive Topics and Reluctant Respondents: Demonstrating a Link between Nonresponse Bias and Measurement Error, in: Public Opinion Quarterly, 74. Jg. (2010), H. 3, S. 413 -432 .

15 Vorausgesetzt, das Rauchverhalten ist unabhängig von der Bereitschaft, an Umfragen teilzunehmen und beide Gruppen - also Raucher und Nichtraucher - neigen nicht in unterschiedlichem Maße zu sozial erwünschtem Antwortverhalten. Einen indirekten Beleg für die Angemessenheit der Annahmen liefert der Befund, dass die Bereitschaft, sich in der zweiten Erhebung nach dem Volksentscheid nochmals befragen zu lassen, sich zwischen Rauchern (45 Prozent) und Nichtrauchen (49 Prozent) nicht nennenswert unterscheidet. 


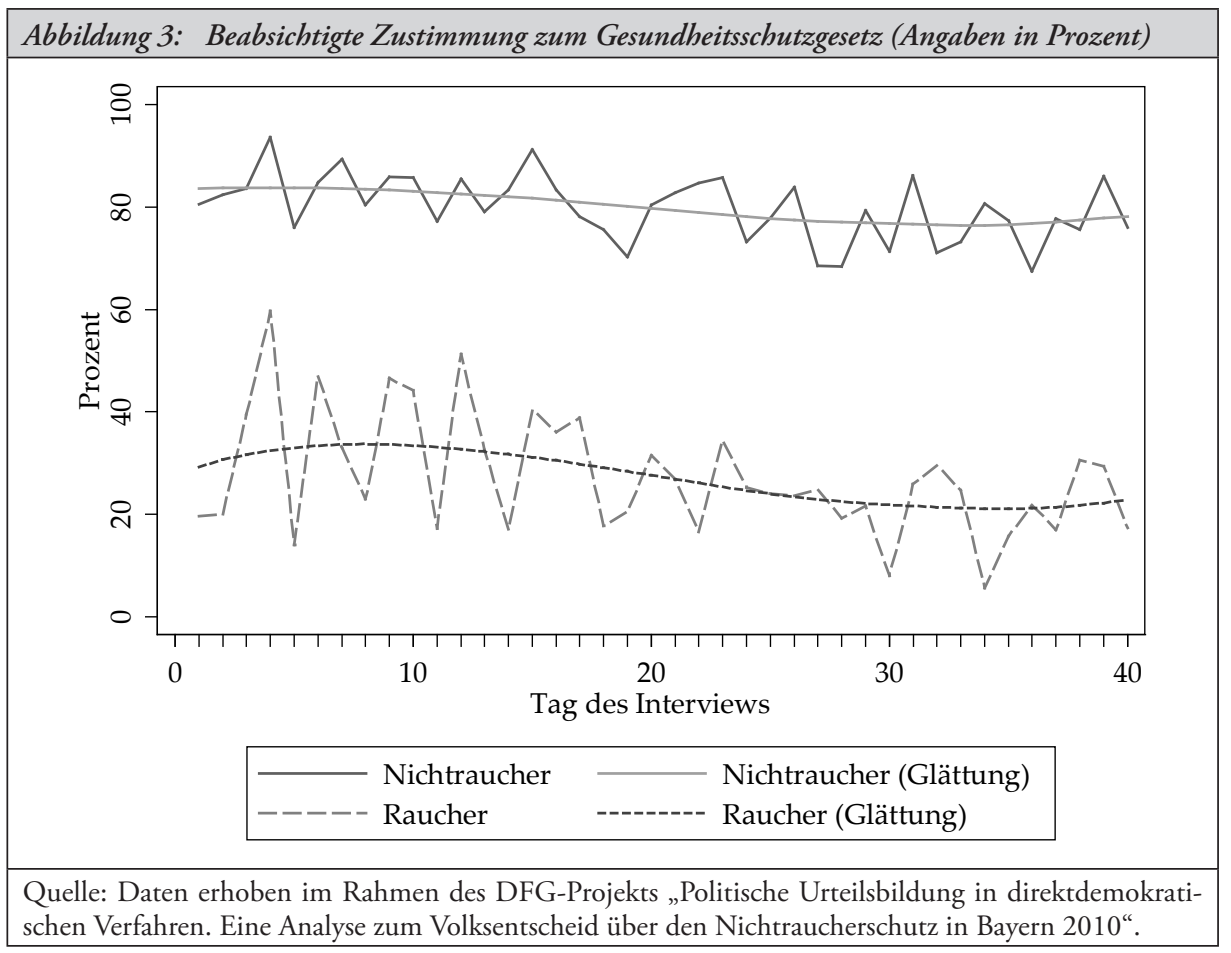

nen, es gehe um eine Entscheidung zwischen individueller Freiheit und staatlicher Intervention. ${ }^{16}$ Würden Nichtraucher diesen Eindruck gewinnen, so die Annahme, würden sie mit Nein stimmen - und so zur Niederlage des Gesetzentwurfes beitragen.

Aus dem Sieg der Befürworter und der Polarisierung des Stimmverhaltens zwischen Rauchern und Nichtrauchern darf man allerdings nicht schließen, Einstellungen zu staatlicher Intervention seien für das Stimmverhalten vollkommen bedeutungslos gewesen. Dies zeigt ein logistisches Regressionsmodell, in dem die Stimmentscheidung mit verschiedenen Faktoren zu erklären versucht wird, darunter der Einstellung zu staatlicher Intervention. Wie Tabelle 1 zu entnehmen ist, geht von dieser Einstellung auch unter Kontrolle anderer Merkmale ein deutlicher Effekt aus. Der statistisch signifikante Regressionskoeffizient steht dabei für einen bedeutsamen Einfluss auf die Wahrscheinlichkeit, beim Volksentscheid mit Ja zu votieren. Hält man alle anderen unabhängigen Variablen am passenden Mittelwert fest und bewegt sich von einer sehr schlechten zu einer sehr guten Bewertung staatlicher

16 Siehe zu theoretischen Grundlagen und empirischen Befunden solchen so genannten Framings Robert M. Entman, Framing: Toward Clarification of a Fractured Paradigm, in: Journal of Communication, 43. Jg. (1993), H. 1, S. 51 - 58; Dennis Chong / James N. Druckman, Framing Public Opinion in Competitive Democracies, in: American Political Science Review, 101. Jg. (2007), H. 4, S. 637 - 655; Thorsten Faas / Harald Schoen, Mehrwertsteuer und Staatsverschuldung: Lassen sich die Einstellungen der Bevölkerung durch Framing verschieben?, in: Thorsten Faas / Kai Arzheimer / Sigrid Roßteutscher (Hrsg.), Information - Wahrnehmung - Emotion: Politische Psychologie in der Wahl- und Einstellungsforschung, Wiesbaden 2010, S. 123 - 143. 
Intervention, nimmt die Wahrscheinlichkeit einer Zustimmung um über 30 Prozentpunkte zu. Die Position gegenüber staatlicher Intervention scheint das Votum also durchaus beeinflusst zu haben. ${ }^{17}$

Allerdings unterschieden sich die Wirkungen auf das letztliche Stimmverhalten von Rauchern und Nichtrauchern. Der in Tabelle 1 dargestellte Einfluss der Einstellung zur Rolle des Staates führt dazu, dass etwa vier Prozent der Raucher, die andernfalls mit Nein stimmen wollten, sich für ein Ja entschieden. Umgekehrt votierten unter dem Einfluss dieser Einstellung rund 14 Prozent der Raucher, die sonst Ja gesagt hätten, mit Nein. Gerade spiegelverkehrt verhält es sich bei den Nichtrauchern. Hier wurden aus rund drei Prozent

\begin{tabular}{|c|c|c|}
\hline Unabhängige Variable & $\mathrm{b}$ & Standardfehler \\
\hline Einstellung zum Nichtraucherschutz & $8.75^{* *}$ & 0.56 \\
\hline Einstellung zur Rolle des Staates & $2.90^{* *}$ & 0.36 \\
\hline Identifikation CDU/CSU & -0.03 & 0.18 \\
\hline Identifikation SPD & -0.38 & 0.21 \\
\hline Identifikation Grüne & -0.17 & 0.30 \\
\hline Identifikation FDP & $-0.70^{*}$ & 0.32 \\
\hline Alter & 0.005 & 0.004 \\
\hline Geschlecht (Frau) & $0.61^{* *}$ & 0.15 \\
\hline Hohe Bildung & 0.21 & 0.26 \\
\hline Konstante & $-4.96^{* *}$ & 0.37 \\
\hline Korrigiertes Pseudo- $\mathrm{R}^{2}$ (McFadden) & 0.42 & \\
\hline $\mathrm{N}$ & 2.579 & \\
\hline \multicolumn{3}{|c|}{$\begin{array}{l}\text { Anmerkung: Ausgewiesen sind unstandardisierte Logitkoeffizienten und Standardfehler. }{ }^{*} \mathrm{p}<.05 \text {, } \\
\text { ** } \mathrm{p}<.01 \text {. } \\
\text { Abhängige Variable: „Beim Volksentscheid am 4. Juli können Sie für oder gegen den Gesetzentwurf „Für } \\
\text { echten Nichtraucherschutz!“ stimmen. Wie werden Sie stimmen: für (1) oder gegen den Gesetzentwurf } \\
\text { (0)?”. Es wurden nur Befragte berücksichtigt, die wahrscheinlich oder bestimmt an dem Volksentscheid } \\
\text { teilnehmen wollten. } \\
\text { Unabhängige Variable: Nichtraucherschutz (Cronbachs } \alpha=0.62) \text { : Mittelwertindex aus den Bewertungen } \\
\text { (fünfstufige Antwortvorgabe von „stimme überhaupt nicht zu“ (0) bis ,stimme voll und ganz zu“ (1)) } \\
\text { der folgenden Aussagen: „Das Problem des Nichtraucherschutzes lässt sich durch Raucherräume lösen.“ } \\
\text { (umgepolt), „In Speiselokalen sollte das Rauchen verboten werden.“, „Rauchen sollte überhaupt verbo- } \\
\text { ten werden.“, „In Festzelten sollte man rauchen dürfen.“ (umgepolt), „In Kneipen sollte rauchen erlaubt } \\
\text { sein.“ (umgepolt). Rolle des Staates (Cronbachs } \alpha=0.53 \text { ): Mittelwertindex aus den Bewertungen (fünf- } \\
\text { stufige Antwortvorgabe von „stimme überhaupt nicht zu“ (0) bis „,stimme voll und ganz zu“ (1)) der } \\
\text { folgenden Aussagen: „Es ist die Pflicht des Staates, Menschen vor gesundheitlichen Risiken zu schüt- } \\
\text { zen.“, „Um die Sicherheit der Bürger zu garantieren, sollte der Staat Freiheitsrechte des Einzelnen ein- } \\
\text { schränken.“, „Der Staat sollte sich nicht in Entscheidungen des Einzelnen darüber einmischen, wie er } \\
\text { sein Leben führt.“ (umgepolt). Parteiidentifikation: Es wurde für die betrachteten Parteien jeweils eine } \\
\text { Dummy-Variable gebildet (1: Identifikation mit der Partei; 0: keine / andere Parteiidentifikation). Alter } \\
\text { in Jahren. Hohe Bildung: } 1 \text { (mindestens Fachabitur), } 0 \text { (andere). } \\
\text { Quelle: Daten erhoben im Rahmen des DFG-Projekts „Politische Urteilsbildung in direktdemokrati- } \\
\text { schen Verfahren. Eine Analyse zum Volksentscheid über den Nichtraucherschutz in Bayern 2010“. }\end{array}$} \\
\hline
\end{tabular}

17 Weiterführende Analysen zeigen, dass der Effekt dieser Einstellungsdimension das individuelle Stimmverhalten von Rauchern etwas stärker beeinflusst als jenes von Nichtrauchern. 


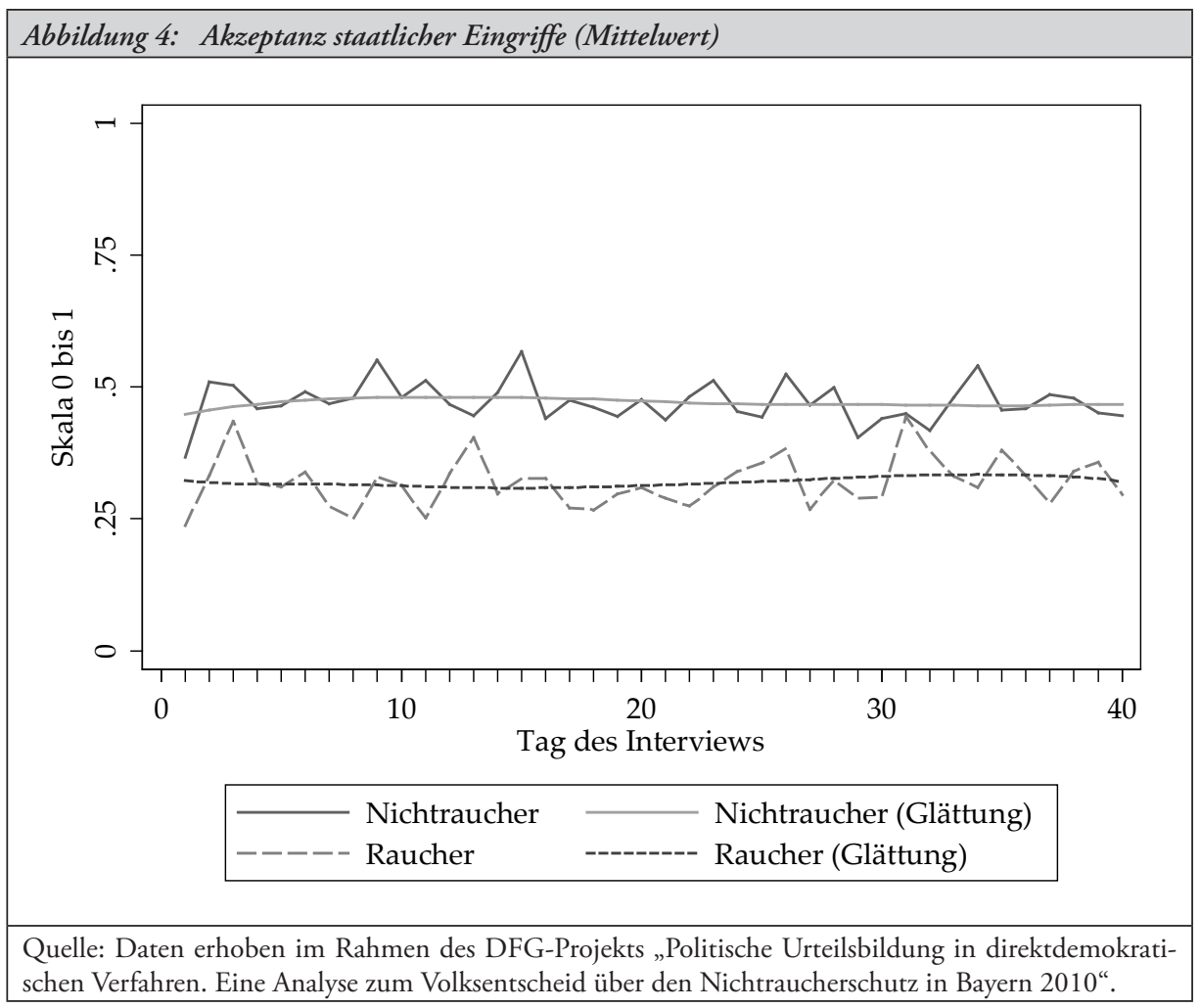

der Ja-Voten Nein-Stimmen, und aus etwa 13 Prozent der Nein-Stimmen wurden Voten für den Gesetzentwurf für ein striktes Rauchverbot. Die Position zur Rolle des Staates sorgte für mehr Nein-Stimmen von Rauchern und mehr Ja-Stimmen von Nichtrauchern. Sie wirkte der oben beobachteten Polarisierung im Stimmverhalten also nicht entgegen, sondern trug zu dieser bei.

Dieses zunächst erstaunliche Ergebnis hat seine Ursache darin, dass sich Raucher und Nichtraucher in ihrer Bewertung staatlicher Intervention unterscheiden. Raucher treten staatlicher Intervention im Durchschnitt skeptisch gegenüber (siehe Abbildung 4), wie dem Mittelwert von rund 0,3 auf einer von 0 bis 1 reichenden Skala zu entnehmen ist. Lediglich 15 Prozent weisen einen Wert oberhalb von 0,5 und somit eine positive Haltung auf. Die Nichtraucher zeigen sich für staatliche Eingriffe zum Schutz des Einzelnen deutlich aufgeschlossener als Raucher, wie sich am Mittelwert von knapp 0,5 ablesen lässt. Einige Nichtraucher betrachten staatliche Intervention skeptisch, wenngleich nur sehr wenige eine außerordentlich kritische Haltung einnehmen; etliche stehen ihr indifferent gegenüber oder befürworten sie. Unter den überwiegend interventionskritischen Rauchern konnte der Einfluss von Einstellungen zu staatlichen Eingriffen vergleichsweise viele Bürger von einem Votum für den Gesetzentwurf abhalten. Das gilt nur für einen kleinen Teil der Nichtraucher. Die zahlreichen Nichtraucher, die staatliche Eingriffe begrüßen oder ihnen indifferent gegenüberstehen, konnten von diesen Einstellungen schwerlich zu einem Votum gegen ein strikteres Rauchverbot bewegt werden. 
Um genügend Nichtraucher auf ihre Seite zu ziehen, hätte den Gegnern des vorgeschlagenen kompletten Rauchverbots also zweierlei gelingen müssen: Sie hätten zum einen ihre Lesart des Volksentscheids als Entscheidung über das fragile Gleichgewicht zwischen staatlichen Eingriffen und individueller Freiheit durchsetzen müssen. Zum anderen hätten sie vielen Nichtrauchern den Eindruck vermitteln müssen, staatliche Eingriffe zum Schutz des Einzelnen seien von Übel. Das erste Ziel konnten sie zum Teil erreichen, wie sich an dem Effekt in Tabelle 1 erkennen lässt. Das zweite Ziel verfehlten sie jedoch weitgehend, denn während der Kampagnenphase blieben die Nichtraucher praktisch unverändert bei ihrer durchschnittlich indifferenten Haltung zu staatlicher Intervention.

\section{Fazit: Initialzündung für den Nichtraucherschutz in Deutschland?}

Beim bayerischen Volksentscheid über den Nichtraucherschutz unterscheiden sich Raucher und Nichtraucher hinsichtlich der Rezeption der Kampagnen, des Interesses am Volksentscheid und der Kenntnisse über den Abstimmungsgegenstand sowie die relevanten Akteure nicht. Dagegen scheinen Nichtraucher mit etwas höherer Wahrscheinlichkeit am Volksentscheid teilgenommen zu haben, der insoweit kaum oder überhaupt nicht eine Frage des Rauchverhaltens war. Dagegen kann der Einfluss des Rauchverhaltens auf die Stimmentscheidung kaum überschätzt werden. Etwa 80 Prozent der Nichtraucher stimmten für die Verschärfung des Rauchverbots, ein beinahe ebenso großer Anteil der Raucher dagegen. Das Stimmverhalten war also entlang dieser Unterscheidung stark polarisiert und entsprach damit den Erwartungen und Wahrnehmungen der Öffentlichkeit. Der Volksentscheid war cum grano salis ein Sieg von Nichtrauchern über Raucher.

Den Gegnern der Gesetzesverschärfung gelang es also nicht, zahlreiche Nichtraucher für ein Nein zu gewinnen. Dieser Befund ist umso bedeutsamer, als die „Bayern sagt nein“Kampagne genau darauf abzielte, die Trennlinie zwischen Rauchern und Nichtrauchern für das Stimmverhalten bedeutungslos zu machen. Dass dieses Ziel nicht erreicht wurde, dürfte zum Teil daran liegen, dass die Nein-Kampagne, ebenso wie die Ja-Kampagne, eine begrenzte öffentliche Resonanz fand. Erschwert wurde die Nein-Kampagne sicherlich auch dadurch, dass sie öffentlich mit schlecht beleumundeten Interessengruppen wie der Tabakindustrie verbunden wurde. Abgesehen von diesen Faktoren waren die Ausgangsbedingungen für die Gegner des strikten Rauchverbots ungünstig. Zum einen scheint es derart naheliegend und intuitiv zu sein, einen Volksentscheid über ein Rauchverbot als Konflikt zwischen Rauchern und Nichtrauchern zu interpretieren ${ }^{18}$, dass es massiver Kampagnen oder gravierender Ereignisse bedürfte, um eine andere Lesart durchzusetzen. Zum anderen stehen die bayerischen Nichtraucher staatlichen Eingriffen offenbar nicht überwiegend skeptisch gegenüber. Selbst wenn sie allein nach ihrer Haltung zu staatlicher Intervention entschieden hätten, hätten sie daher nicht zwangsläufig geschlossen gegen ein Rauchverbot votiert. Zusammengenommen scheinen die Gegner des strikten Rauchverbots einen Kampf geführt zu haben, den sie kaum gewinnen konnten.

18 Siehe auch Donald Philip Green / Ann Elizabeth Gerken, Self-interest and Public Opinion Toward Smoking Restrictions and Cigarette Taxes, in: Public Opinion Quarterly, 53. Jg. (1989), H. 1, S. $1-16$. 
Diese Diagnose legt die Folgerung nahe, dass ein neuerlicher Volksentscheid über das Rauchverbot in Bayern zum substantiell gleichen Ergebnis wie jener am 4. Juli 2010 führen würde. Eine Lockerung des Verbots durch eine parlamentarische Mehrheit dürfte daher ebensowenig Bestand haben, wie ein Volksbegehren zugunsten einer Liberalisierung realistische Erfolgsaussichten hat. ${ }^{19}$ Auch wenn mit der neuen Regelung nicht alle Bürger Bayerns zufrieden sind, dürfte der Volksentscheid insoweit tatsächlich, wie etwa von Ministerpräsident Horst Seehofer und Landesgesundheitsminister Markus Söder behauptet, eine befriedende Wirkung entfalten. ${ }^{20}$ Mit einer Revision wäre demnach allenfalls dann zu rechnen, wenn sich eine andere Interpretation von Rauchverboten als die bisher vorherrschende und offenbar intuitive durchsetzte und die Bürger in dieser Hinsicht eine verbotskritische Haltung einnähmen.

Scheint mit dem Volksentscheid vom Juli 2010 die Auseinandersetzung um Rauchverbote in Bayern beendet, so muss dies nicht für die gesamte Bundesrepublik gelten. Vielmehr könnten die bayerischen Erfahrungen mit direktdemokratischen Verfahren zur Regelung des Nichtraucherschutzes andernorts als Vorbild dienen. Auch dort sind die Nichtraucher in der Mehrheit. ${ }^{21}$ Wenn bei der Entscheidung über eine Verschärfung von Rauchverboten das eigene Rauchverhalten so wirkmächtig ist, könnten Nichtraucher daher den direktdemokratischen Weg beschreiten, um striktere Regeln für den Nichtraucherschutz durchzusetzen. Der Volksentscheid am 4. Juli 2010 könnte somit einen politischen Konflikt in Bayern beendet haben, zugleich aber zum ersten Kapitel in einer längeren Geschichte über direktdemokratische Verfahren und den Nichtraucherschutz in Deutschland werden.

19 In einer Nachbefragung von rund der Hälfte der 4.000 vor dem Volksentscheid befragten Personen sprach sich eine deutliche Mehrheit von 63 Prozent gegen ein solches Volksbegehren aus. Nichtraucher traten mit großer Mehrheit gegen eine erneute Abstimmung ein (73 Prozent), während sich bei den Rauchern Zustimmung (40 Prozent) und Ablehnung (39 Prozent) die Waage hielten.

20 Vgl. Birgit Kruse / Mike Szymanski, Interview mit Markus Söder, in: Süddeutsche-Online vom 20. September 2010, http://www.sueddeutsche.de/bayern/markus-soeder-auf-der-wiesn-dasrauchverbot-funktioniert-ich-sehe-keine-exzesse-1.1002058 (Abruf am 25. August 2011).

21 Vgl. Robert Koch-Institut, Daten und Fakten: Ergebnisse der Studie „Gesundheit in Deutschland aktuell 2009“, Berlin 2010, S. 94. 